\title{
Developing Best Practices for Air Quality Sensor Deployments Through Testing
}

\author{
Christine Kendrick $\dagger$ \\ Bureau of Planning and \\ Sustainability \\ City of Portland \\ Portland, OR, USA \\ christine.kendrick@port \\ landoregon.gov
}

\author{
Devin Wilde \\ Environmental Science \\ and Management \\ Portland State \\ University \\ Portland, OR, USA \\ dwilde@pdx.edu
}

\author{
Kevin Martin \\ Bureau of Planning and \\ Sustainability \\ City of Portland \\ Portland, OR, USA \\ kevin.martin@portlando \\ regon.gov
}

\author{
Linda George \\ Environmental Science \\ and Management \\ Portland State \\ University \\ Portland, OR, USA \\ georgel@pdx.edu
}

\begin{abstract}
Lower cost air quality sensors have the potential to provide air pollution information at higher spatial resolutions than traditional monitoring. Such information could be used to evaluate project and planning impacts plus disparities in air pollution health impacts across populations. However, lower cost air quality sensors have technical limitations, hardware sustainability considerations, and resource issues associated with deploying and managing a network in the public right of way. To help improve understanding about the uses and limitations, the City of Portland Bureau of Planning and Sustainability tested three types of low cost air quality sensor devices across various field and lab deployments in collaboration. The project supported multi-disciplinary partnership development between the City, Oregon Department of Environmental Quality (DEQ), Portland State University (PSU) researchers, Green Electronics Council (GEC) and multiple private sector partners. This paper details findings about sensor performance in various deployment configurations. Results highlight challenges and recommendations that can be used to improve interoperability for air quality sensor networks. Findings about maintenance planning and sensor sustainability to minimize electronics waste are also discussed. Through this testing design, it was established that lower cost air quality sensors are capable of measuring local roadside pollution signals in addition to urban background pollution. Establishing this pattern is key to using sensors to support additional planning and assessment questions.
\end{abstract}

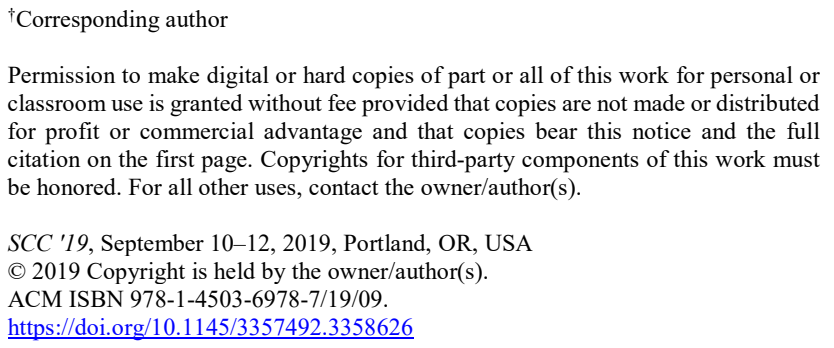

Permission to make digital or hard copies of part or all of this work for personal or classroom use is granted without fee provided that copies are not made or distributed for profit or commercial advantage and that copies bear this notice and the full citation on the first page. Copyrights for third-party components of this work must be honored. For all other uses, contact the owner/author(s).

SCC '19, September 10-12, 2019, Portland, OR, USA (C) 2019 Copyright is held by the owner/author(s). ACM ISBN 978-1-4503-6978-7/19/09. https://doi.org/10.1145/3357492.3358626

This work is licensed under a Creative Commons Attribution-NonCommercialNoDerivs International 4.0 License.

\section{CCS CONCEPTS}

- Sensor applications and deployments - Sensor devices and platforms

\section{KEYWORDS}

Smart City, Internet of Things, Air Quality, Sensors

\section{ACM Reference format:}

Christine Kendrick, Devin Wilde, Kevin Martin and Linda George. 2019. Developing Best Practices for Air Quality Sensor Deployments Through Testing. In Proceedings of the 2nd ACM/EIGSCC Symposium On Smart Cities and Communities (SCC 2019). ACM, New York, NY, USA. https://doi.org/10.1145/3357492.3358626

\section{Introduction}

Lower cost air quality sensors have the potential to provide air pollution information at higher spatial resolutions than traditional monitoring. Such information could be used to make more datadriven decisions about mobility project impacts, policies to mitigate health effects of pollution, and understand inequities in air pollution exposure. Lower cost sensors may also help to fill in data gaps for smaller cities or undeveloped regions without traditional monitoring sites [1]. Additional spatially- and temporally-resolved data could be used to improve air quality models, pollutant inventories, estimates of personal exposure, comparison to satellite remote sensing data, and support for emergency response needs and activation of hazard-warning systems [2], [3].

However, lower cost air quality sensors have technical limitations, hardware sustainability considerations, and resource issues associated with deploying and managing a network in the public right of way. Sensitivity, stability, and longevity of operation before replacement are continued technological challenges in lower cost air sensors [3], [4]. Most sensor studies assess performance under a range of specific conditions or how the sensor fits a specific purpose [1], [4]. There is a need for continued documentation of performance in the field, over long-term periods, and in varying 
pollution environments. Without additional documentation of device or sensor platforms in these configurations, it is difficult for a user to know if the sensor platform is sufficient for the intended purpose when acquiring such devices [1].

City of Portland Bureau of Planning and Sustainability (BPS) tested three types of low cost air quality sensor devices in various field and lab deployments. The project supported multi-disciplinary partnership between the City, Oregon Department of Environmental Quality (DEQ), Portland State University (PSU), Green Electronics Council (GEC) and private sector partners. This paper details findings about sensor performance in the deployment configurations to improve understanding about use and limitations.

\section{Methods}

\subsection{Sensor Selection}

To select which sensors would be tested in the various deployment phases, a unique sensor review process was created and conducted. Initial review criteria were assessed for forty-three low-cost sensors/devices and a second round of review was conducted for seven devices in the fall of 2016. Multiple sensor devices were selected to evaluate different device setups and data models. Nine devices total were tested, three from each provider:

- $\quad$ Argonne National Laboratory \& University of Chicago Array of Things (AoT) Nodes

- $\quad$ SenSevere RAMPs

- $\quad$ Apis SensorCell Nodes

The AoT is a multi-sensor platform designed to provide greater spatial and temporal resolution of health-related variables in cities; multiple microsensors measure airborne particles and an array of pollutants in one unit with open-sourced data [5]. The nodes are designed to operate with little to no maintenance once connected to power. Data is transmitted wirelessly via cellular modem to Argonne's Beehive. Data from every sensor is available in a csv file. Python scripts created by the AoT team are used to process data to air pollutant concentrations. AoT air quality measurements discussed here include $\mathrm{CO}, \mathrm{NO}_{2}$, and $\mathrm{O}_{3}$ from Screen Printed ElectroChemical (SPEC) sensors placed on circuit boards inside the AoT housing. $\mathrm{PM}_{2.5}$ is measured by the Alphasense Optical Particle Counter OPC-N2 placed at the top of the AoT housing and has an active fan to sample air within the OPC-N2.

The SenSevere Real-time, Affordable, Multi-Pollutant (RAMP) was developed in partnership by Carnegie Mellon University's Center for Atmospheric Particles and a Pittsburgh start-up, SenSevere. The SenSevere RAMPs use a random forest machine learning method for calibration built from field deployment data [6]. Data is transmitted wirelessly via cellular modem. Data is processed and fitted through random forest calibration model using a combination of python and R scripts. RAMP air quality measurements discussed here include $\mathrm{CO}, \mathrm{NO}_{2}$, and $\mathrm{O}_{3}$ from
Alphasense electrochemical sensors. Sensors use passive sampling open to the air through the bottom of the RAMP housing.

The Apis SensorCell node measures multiple pollutants from an active sampling inlet and includes an aspirated shield design to cool down surface heating and internal electronics. SensorCell nodes are calibrated by Apis to Federally Equivalent Method (FEM) standards and known concentrations of gases at various temperature and relative humidity ranges. Proprietary data processing algorithms are used to post-process data. Data is transmitted wirelessly via cellular modem and stored in Apis's cloud hosting service and can be exported as a csv or accessed via Application programming interface (API). Apis air quality measurements discussed here include $\mathrm{CO}, \mathrm{NO}, \mathrm{NO}_{2}$, and $\mathrm{O}_{3}$ from Alphasense electrochemical sensors.

Sensor selection can be a difficult process for cities and agencies to navigate. There are many decision points that require a multidisciplinary team to evaluate which sensor/device package fits project needs. Lower cost and distributed sensors also differ from typical infrastructure and technology procurements that government agencies have worked with historically. Many of the solution providers are start-up companies themselves, the devices will not last as long as other types of hardware, and most products will have a unique software or data processing component. Lessons learned from the sensor selection process and procurement coordination developed for this project were summarized and incorporated into Section 2.3 and Section 2.6 of the Global City Teams Challenge (GCTC) Blueprint document titled Recommendations for the Development and Implementation of Distributed Sensor Networks [7].

Through partnership with Green Electronics Council (GEC), the City of Portland was able to specifically incorporate the consideration of electronics waste into the procurement criteria. By adding a requirement that the device can be upgraded or modified, the potential to reduce electronics waste is introduced. In this grant project, the addition of that procurement criteria resulted in a sensor design change for one of the vendors (Apis, Inc) that was part of the project. A reusable, modular socket design for the gas sensors was kept in final SensorCell design. The modular socket is a cost saving measure and leads to less consumption of printed circuit boards (PCBs). PCB production results in hazardous solid waste (tin/lead dross, hole drilling dust, etc.) and liquid waste (solutions for acid etching, rack and lead stripping, plated through hole (PTH) copper, etc.).

\subsection{Lab Deployment}

PSU researchers in the Sustainable Atmospheres Research (STAR) lab evaluated the performance of the AoT nodes for $\mathrm{PM}_{2.5}$ in laboratory conditions. The three AoT nodes initially received were moved through extensive debugging processes by PSU and Argonne National Laboratory researchers. In order to upload node data to Argonne's Beehive, cellular connectivity must be maintained. The nodes were not reliably maintaining the cellular 
Developing Best Practices for Air Quality Sensor Deployments Through Testing

signal in the PSU lab, so modifications were made to bypass the cellular modem and connect the nodes directly to the internet via a switch and local LAN port. This modification did not resolve the problem on one of the nodes which had to be sent back to Argonne National Laboratory for replacement. Due to these limitations, only Nodes 64 and 65 were evaluated for $\mathrm{PM}_{2.5}$ performance in the lab deployment.

Lab testing included both chamber and ambient testing. AoT Nodes 64 and 65 were placed inside a jacketed stainless-steel testing chamber and exposed to particulate matter lit from a match and placed in a jar inside the chamber. This PM source was found to be more reliable than the use of polycyclic latex spheres and concentration ramping due to lack of stable outputs from instrument. An FEM DustTrak Model 8533 instrument sampled the same air from the chamber. Each chamber test took from 3 hours to 4 hours while the smoke dissipated and reached zero concentration. All tests occurred at $17^{\circ} \mathrm{C}\left(75^{\text {th }}\right.$ percentile of Portland ambient temperatures), consistently controlled using the chamber watercooling system.

Outdoor ambient testing occurred on the roof of the PSU STAR lab building. A FEM DustTrak was installed outside alongside the AoT nodes to sample ambient air for approximately 4 days. Linear regression was used to compare and analyze the results from the laboratory chamber tests $(n=10)$ and the outdoor ambient tests (approximately 4 days of continuous testing).

\subsection{DEQ Field Deployments}

Devices were deployed at the Oregon Department of Environmental Quality (DEQ) SE Lafayette urban background station so that sensor measurements could be compared to Federal Reference Method (FRM) instruments in outdoor ambient air. This field setup also allowed for comparisons of devices of the same type to each other. To accommodate scheduling conflicts throughout the study period due to procurement and manufacturing delays and lab availability, each device type was deployed in sets of threes to the DEQ SE Layfette station site for different periods. Devices were deployed to the top deck of the station where inlets for reference DEQ instruments are located. FRM data was obtained from DEQ.

Apis co-location period occurred for approximately one month through December 2016 to January 2017. SenSevere devices were deployed from August 2018 through April 2018. The co-location period of the SenSevere RAMPS at the DEQ station was much longer than the Apis co-location period ( 8 months versus 1 month). This was due to the difference in calibration methods and forest fire events in the Portland area. The Apis devices are calibrated by Apis through building calibration equations when comparing the sensors to known concentrations of gases at various temperature and relative humidity ranges. The first 4 months of the co-location of the RAMPS at the DEQ station were used to build and train the random forest models. This period included some forest fire events,
SCC 2019, September, 2019, Portland, Oregon, USA

so it was extended beyond the Zimmerman et al. [6] recommended period to cover a better range of expected ambient conditions.

AoT co-location at the DEQ site occurred from May 2019 to June 2019 for Nodes 64, 65, and 7. Node 7 was received later due to the connectivity debugging issue described in Section 2.2. This period also occurred later than other deployments due to manufacturing delays and connectivity and node replacements described in Section 2.2. PSU STAR lab researchers conducted this field testing for the City of Portland. Temperature measurements from AoT Nodes were also compared to DEQ temperature measurements for this deployment. Over the study month, brief power outages led to some nodes not operating for several days. Results presented are for periods in which all three nodes uploaded $100 \%$ of their data.

\subsection{Roadside Field Deployment}

From May 2018 through the end of the study period Dec 2018 presented here, the Apis SensorCell nodes and RAMPs were deployed on traffic signal poles at three intersections along the SE $122^{\text {nd }}$ Ave corridor, which is a major north/south urban arterial in Portland, OR. The roadside deployment sites were at the intersection with SE Division, SE Market and SE Stark. Evening peak hour traffic flows from 2017 for each study intersection are listed below [8]:

- $\quad 4767$ vehicles per hour for Stark \& $122^{\text {nd }}$ Ave

- 2365 vehicles per hour for Market \& $122^{\text {nd }}$ Ave

- 4745 vehicles per hour for Division \& $122^{\text {nd }}$ Ave

The three intersection sites were chosen due to interest in better understanding air quality along this major corridor. Additional investments in traffic safety sensors, increasing pedestrian and bicyclist facilities, and altering bus routes are also planned or occurring for the corridor. Space for equipment on the poles and cabinets for powering the devices were also considerations.

Despite the field testing at DEQ beforehand and ensuring the sensors were operating continuously, there were multiple instances of equipment or data communications failure throughout the roadside deployment period. Each time a device needed to be modified or have a component replaced, Portland Bureau of Transportation (PBOT) electricians and equipment had to be used to take down the sensor which was resource intensive (manpower, limited availability of time). Data for both devices types was not continuous at each intersection throughout May-December 2018.

\section{Results and Discussion}

\subsection{AoT Lab Test Findings}

In both chamber and outdoor ambient testing, the AoT nodes underestimated $\mathrm{PM}_{2.5}$ concentrations consistently compared to the FEM Dusttrak measurements (Figure 1 and 2). Figure 1 shows linear regression results for 3 out of 10 chamber tests comparing 
the AoT nodes to the FEM DustTrak. For each chamber test, AoT nodes underestimated $\mathrm{PM}_{2.5}$. High r-squared values were present in the regression analysis showing correlation. For all tests conducted, Node 64 estimates concentrations below that of Node 65, highlighting the potential for inter-sensor variation in measuring $\mathrm{PM}_{2.5}$.

During lab rooftop outdoor ambient testing, both the devices and FEM instrument showed diurnal fluctuations in PM. In the top panel of Figure 2, DustTrak measurements show values up to 21 $\mu \mathrm{g} / \mathrm{m}^{3}$ while Node 65 measurements max out at $13 \mu \mathrm{g} / \mathrm{m}^{3}$. In the bottom panel of Figure 2, the inter-sensor variability between Node 64 and 65 is very evident. Both AoT nodes underestimate PM compared to the DustTrak, with Node 64 measuring values below that of Node 65 .

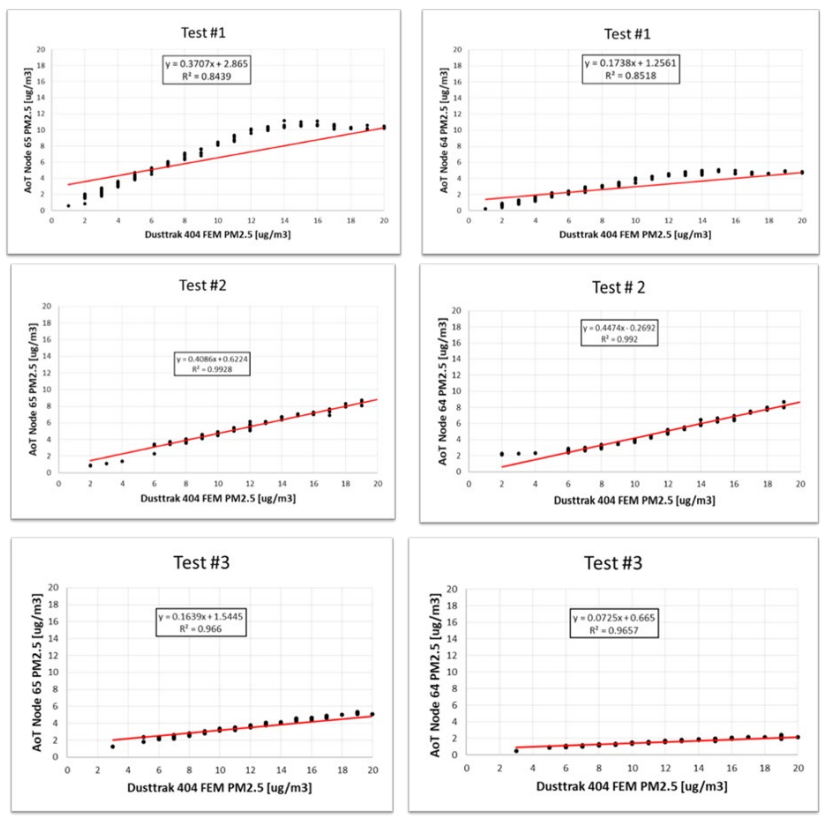

Figure 1: Linear regression results for 3 out of 10 chamber tests comparing AoT Nodes 64 and 65 to FEM DustTrak. Values were recorded beyond $20 \mu \mathrm{g} / \mathrm{m}^{3}$ but analysis truncated to reflect ambient Portland $\mathrm{PM}_{2.5}$ range $\left(0.2 \mu \mathrm{g} / \mathrm{m}^{3}-15 \mu \mathrm{g} / \mathrm{m}^{3}\right)$.

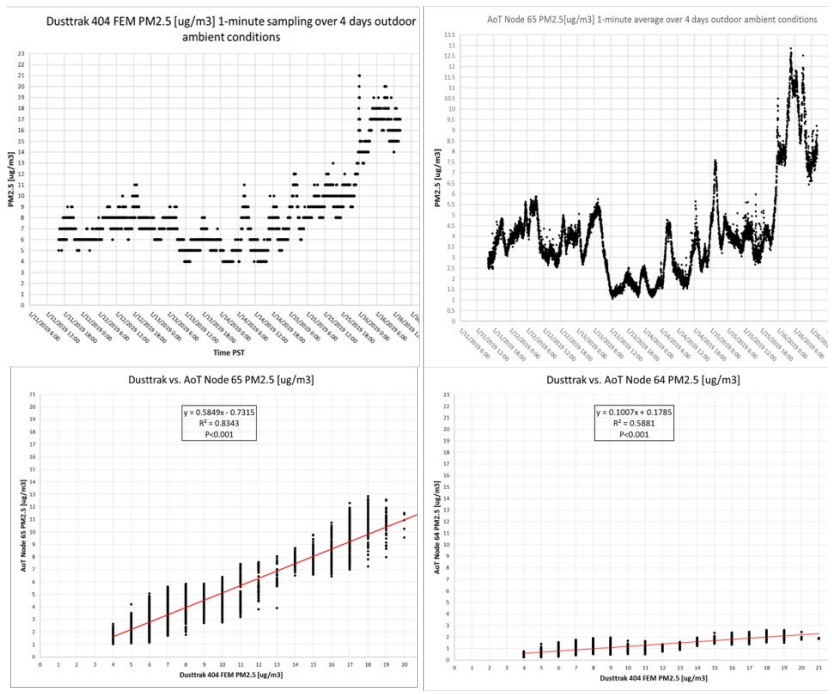

Figure 2: Top panel shows $\mathrm{PM}_{2.5}$ values for the DustTrak and AoT Node 64 during ambient monitoring. Bottom panels compare the AoT Nodes versus DustTrak values

\subsection{DEQ Field Deployment Findings}

Figure 3 shows overall good agreement between the Apis SensorCell data and the DEQ FRM measurements during the colocation period at the SE Lafayette. Correlation coefficients for NO (Figure 3a) range from 0.95 to 0.97 , for $\mathrm{NO}_{2}$ (Figure 3b) 0.89 to 0.96 , for $\mathrm{O}_{3}$ (Figure $3 \mathrm{c}$ ) from 0.95 to 0.98 , and for $\mathrm{CO}$ (Figure $3 \mathrm{~d}$ ) 0.72 to 0.95 . CO concentrations overall are very low in the Portland ambient air so measurement sensitivity will be limited.
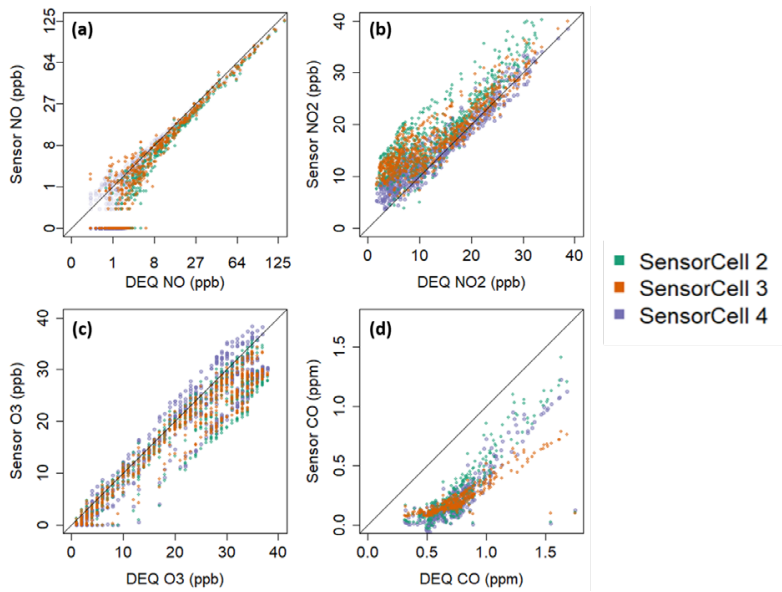

- SensorCell 4

Figure 3: Apis SensorCell vs DEQ FRM measurements during SE Lafayette station co-location for (a) $\mathrm{NO}$ (b) $\mathrm{NO}_{2}$ (c) $\mathrm{O}_{3}$ (d) $\mathrm{CO}$

Figure 4 shows the correlations of the RAMP devices versus the DEQ FRM measurements after the calibration models were built. Correlation coefficients for $\mathrm{NO}_{2}$ (Figure 4a) range from 0.6 to 0.91 , 
Developing Best Practices for Air Quality Sensor Deployments Through Testing

for $\mathrm{O}_{3}$ (Figure 4b) from 0.6 to 0.95 , and for $\mathrm{CO}$ (Figure 4c) 0.12 to 0.99 . The $\mathrm{CO}$ and $\mathrm{O}_{3}$ sensors in RAMP 171 had to be replaced at the end of this deployment. With those values removed, correlation coefficients are 0.84 and 0.91 for $\mathrm{NO}_{2}, 0.84$ and 0.95 for $\mathrm{O}_{3}$, and 0.97 and 0.99 for $\mathrm{CO}$.
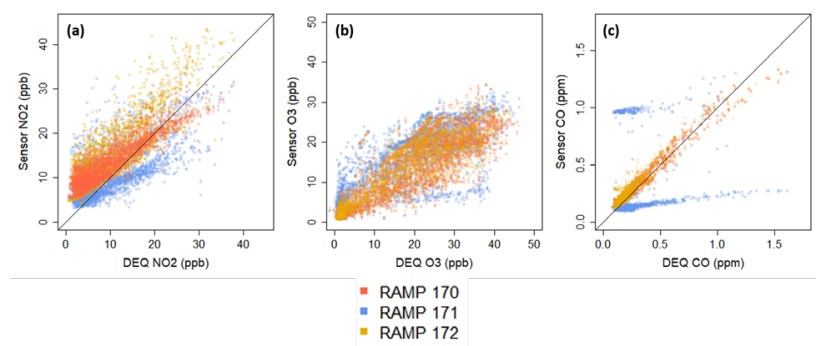

Figure 4: SenSevere RAMP vs DEQ FRM measurements during SE Lafayette station co-location for (a) $\mathrm{NO}_{2}$ (b) $\mathrm{O}_{3}$ (c) $\mathrm{CO}$

Both devices showed overall good agreement with reference instruments. The SenSevere RAMP calibration model required considerably more time deployed at the field station and the values used to convert the electronic signals from the sensors to concentrations are directly tied to the background reference data. Additionally, this deployment phase documented the potential for sensors and electronics components needing to be replaced under six months of use. The devices were easily accessible while deployed at the DEQ station, so replacements were easily coordinated between City staff and the senor manufacturers.

Linear regression results show moderate to weak relationships for all gaseous pollutants when the AoT Nodes are compared to DEQ FRM measurements during co-location. $\mathrm{CO}$ values reported by all three AoT Nodes were negative and showed diurnal patterns with highest values in mid-afternoon. Values below zero are possible since sensors are reporting a change in voltage and then a function is applied to convert that voltage to an air quality concentration. Voltage changes can occur due to temperature, humidity or other pollutant changes and reactions. Manufacturer calibrations can also differ from field conditions so when data is processed zero or negative values are possible. Low ambient $\mathrm{CO}$ concentrations may explain the negative values combined with the lower detection limit of the SPEC CO sensor being $0.5 \mathrm{ppm} . \mathrm{O}_{3}$ results show no relationship between the AoT nodes and DEQ FRM values with all three r-squared values close to zero.

AoT $\mathrm{NO}_{2}$ results show a weak, negative correlation with the DEQ FRM measurements during co-location (Figure 5). Node 64 showed the lowest correlation $\left(r^{2}=0.07\right)$. Figure 5 also shows inconsistent patterns across the AoT Nodes with DEQ FRM values. Node 64 reports values above DEQ FRM values while Nodes 65 and 7 tend to underreport.
SCC 2019, September, 2019, Portland, Oregon, USA

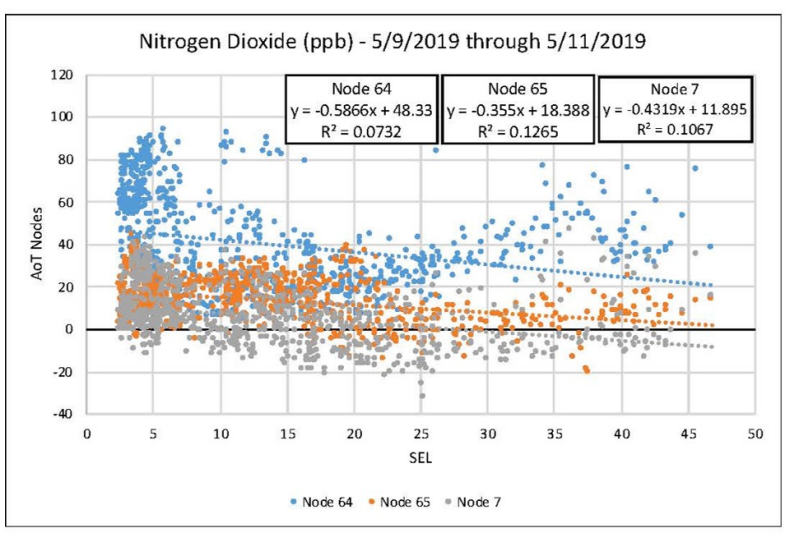

Figure 5: Linear regression for $\mathrm{NO}_{2}$ AoT measurements versus DEQ FRM measurements during SE Lafayette co-location

$\mathrm{PM}_{2.5}$ concentrations during this co-location period had a wider range compared to the gaseous pollutants. Figure 6 shows regression results for two different periods with $100 \%$ data reported and higher and lower PM 2.5 ambient conditions. Both time periods show positive correlations between the AoT Nodes and DEQ FRM measurements. During higher $\mathrm{PM}_{2.5}$ conditions $\left(5 \mu \mathrm{g} / \mathrm{m}^{3}\right.$ to $35 \mu \mathrm{g} / \mathrm{m}^{3}$ ), r-squared values ranges from 0.75 to 0.78 . During lower $\mathrm{PM}_{2.5}$ conditions $\left(2 \mu \mathrm{g} / \mathrm{m}^{3}\right.$ to $\left.8 \mu \mathrm{g} / \mathrm{m}^{3}\right)$, r-squared values ranges from 0.23 to 0.31 .
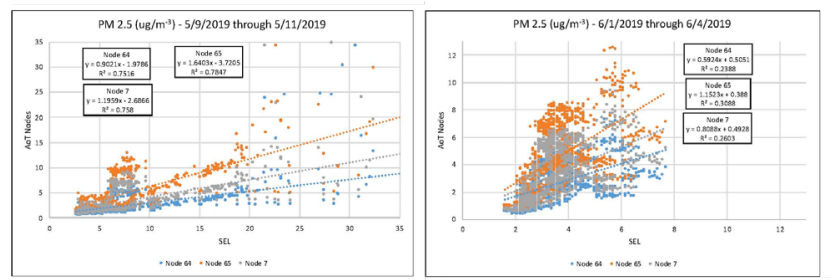

Figure 6: Linear regression for $\mathrm{PM}_{2.5}$ AoT measurements versus DEQ FRM measurements during SE Lafayette co-location for two periods with differing $\mathrm{PM}_{2.5}$ ranges

Temperature values measured from all three AoT Nodes showed strong positive relationship with DEQ temperature measurements (r-squared values ranging from 0.89 to 0.94 ). Node temperature values consistently overestimated the temperature which could be attributed to radiant heat from the AoT nodes or the differences in the DEQ probe being mounted higher on a meteorological tower. Overall, the AoT node temperature sensors were found to be accurate compared to the DEQ measurements.

\subsection{Roadside Deployment Findings}

3.3.1 Local Signal Versus Roadside Signal. When the sensor devices are installed at the roadside, there are limited methods to evaluate performance since there is no reference pollution data at 
the roadside. Air quality sensor devices should be measuring both local effects from the road as well as background pollution levels.

Figure 7 is a thought exercise with simulated data to explore the problem. When the sensor is located right next to the reference instrument, the relationship should be highly correlated with low variability (small spread) (Figure 7a). When the sensor is installed at a location further away from the reference instrument and is compared to the reference data during that period, one should see some correlation but the relationship has more variability. Only part of the sensor data is explained by the background pollution measurement, and the rest theoretically should be attributed to local conditions (Figure $7 \mathrm{~b}$ ). This thought exercise in the data analysis leads us to our first method to be able to explore the roadside data.
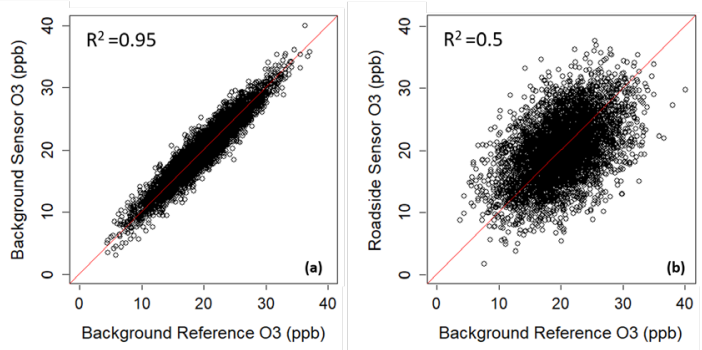

Figure 7: Simulated data for expected results and data analysis method development of roadside sensor performance. (a) Expected relationship when a sensor is co-located with background reference instrument (b) Expected relationship when sensor is located on a roadside away from the background reference instrument

Figure 8 and Figure 9 show this simulated pattern to be true for both devices. The top rows in each figure show sensor measurements versus the background measurements for May 2018 to Dec 2018. The bottom rows show the same relationships for each sensor during the DEQ co-located deployments with less variability and stronger correlations. These patterns begin to indicate that the devices are measuring some type of local signal at the road. However, Figure 8 and Figure 9 also begin to show differences between the two sensor devices despite being co-located on the same traffic signal poles.
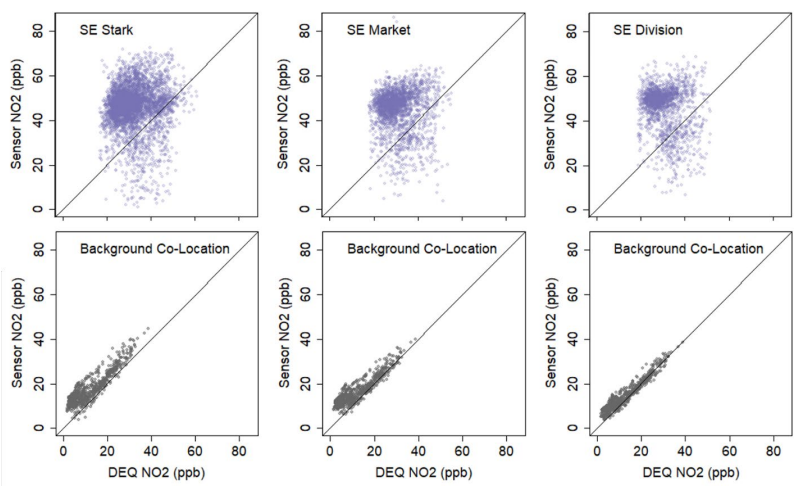

Figure 8: Apis $\mathrm{NO}_{2}$ vs DEQ $\mathrm{NO}_{2}$ measurements during (Top) roadside deployment and (bottom) co-located DEQ deployment
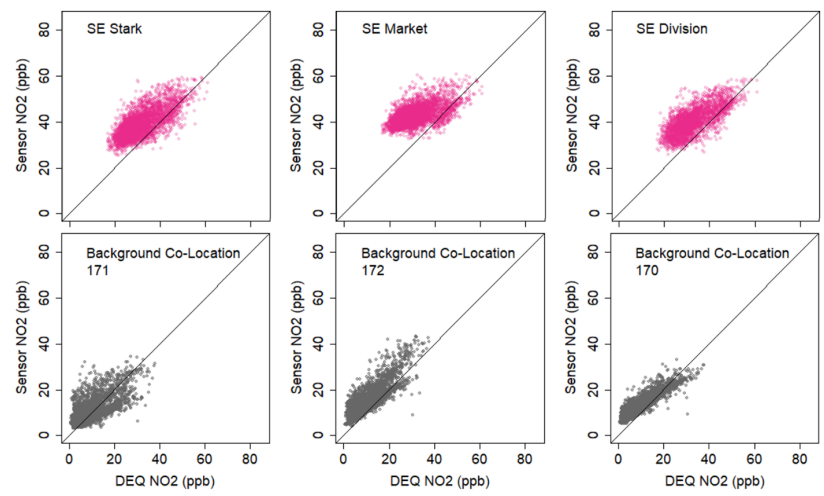

Figure 9: RAMP $\mathrm{NO}_{2}$ vs DEQ $\mathrm{NO}_{2}$ measurements during (Top) roadside deployment and (Bottom) co-located DEQ deployment

To further explore if and how the sensor devices are measuring local pollution impacts at the road, Figure 10 a-c shows the distribution of DEQ FRM and sensor measurements for each device, intersection and pollutant, respectively. For $\mathrm{CO}$, the relationships are different across each intersection (Figure 10a). Ambient CO concentrations are low in Portland, so the signal is difficult to measure and there is not a lot of spatial variation. Differences in CO here are likely due to measurements occurring at the lower end of detection for the sensors. 
Developing Best Practices for Air Quality Sensor Deployments Through Testing

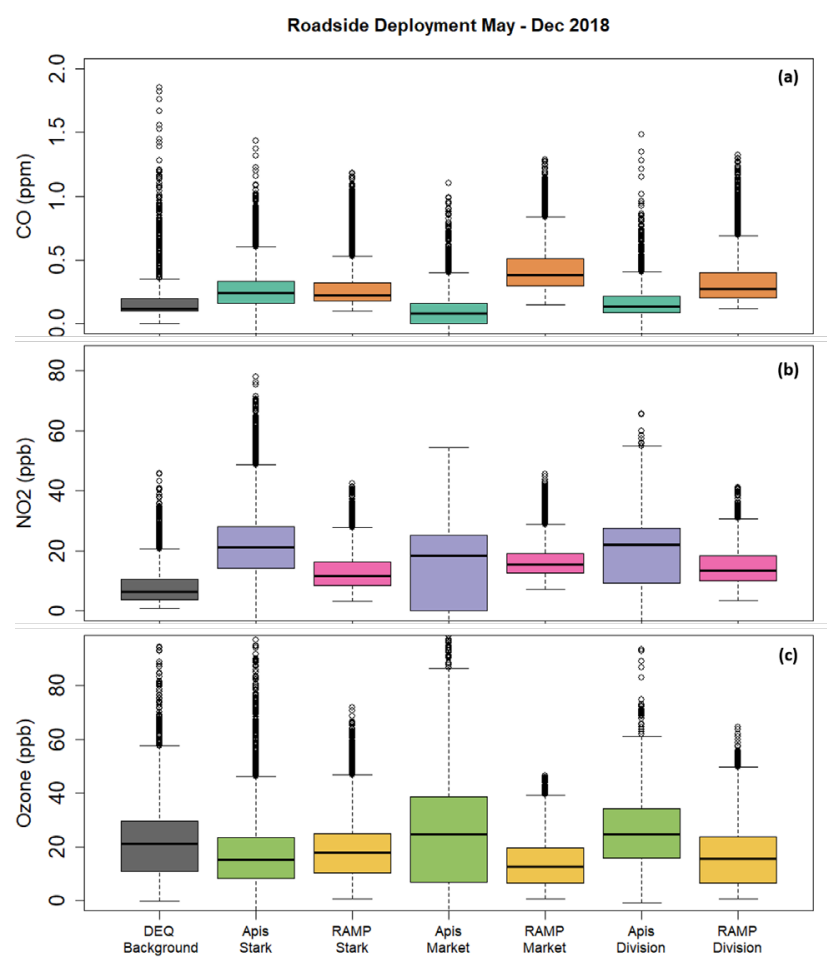

Figure 10: DEQ FRM, Apis, and RAMP distributions during May to Dec 2018 for each intersection for (a) $\mathrm{CO}$ (b) $\mathrm{O}_{3}$ (c) $\mathrm{NO}_{2}$

For $\mathrm{O}_{3}$ distributions, the sensor devices measurements overlap with the background measurements from the same period (Figure 10b). This is expected since ozone is a secondary pollutant and will not necessarily be higher directly within the roadway environment. At times when the sensors are downwind of the intersection depending on wind direction, the ozone could also be lower than the DEQ site due to $\mathrm{NO}$ titration of $\mathrm{O}_{3}$.

For $\mathrm{NO}_{2}$, the roadside measurements for both devices are higher than the measurements at the background site showing the sensors are responding to a local roadway signal (Figure $10 \mathrm{c}$ ). $\mathrm{NO}_{2}$ can have more localized spatial-temporal patterns and can show a relationship with traffic volumes under certain conditions [9].

Figure 10 shows differences between the sensor devices. Apis $\mathrm{NO}_{2}$ median, $75^{\text {th }}$, and $95^{\text {th }}$ percentile values are higher for the Stark and Division intersections compared to Market. This pattern is expected due to the differences in traffic volumes from the cross-streets with $122^{\text {nd }}$ Ave. However, the RAMP $\mathrm{NO}_{2}$ concentrations do not show as much variability across the three intersections.

The exploratory analyses summarized in Figure 10 begin to show both sensor devices are measuring local impacts and regional effects on gaseous air pollutants measured. This preliminary analysis also appears to show the Apis SensorCell nodes are
SCC 2019, September, 2019, Portland, Oregon, USA

measuring more of a local signal compared to the RAMPs. Correlations for the RAMP measurements are stronger for the background data compared to the Apis and background data relationships. Since the RAMPs used the background data in a random forest modeling method to calibrate the sensors, this makes it difficult to unpack the differences. Additionally, the Apis $\mathrm{NO}_{2}$ data tracks with local traffic volumes (higher at Stark and Division intersections compared to Market) while the RAMP data differences across sites do not reflect these patterns.

3.3.2 Sensor Differences. Throughout the roadside deployment period, the measurements between the RAMPs and the Apis nodes are not the same. Most low-cost air quality sensor deployments focus on only one type of sensor device. However, sensors are proliferating and being deployed by various groups within the same area. The issue of how to integrate measurements across devices is something that many cities and agencies will be facing soon. The findings here highlight the observed differences for $\mathrm{NO}_{2}$, and $\mathrm{O}_{3}$.

Figures 11- 14 show an exploratory analysis for $\mathrm{NO}_{2}$ values from the sensor devices. Changes in offsets from zero can be seen for both the Apis and RAMP devices throughout the full period (Figure 11). For each intersection, the $\mathrm{NO}_{2}$ Apis values are generally higher than the RAMP values (Figure 12). This pattern of higher Apis measurements is consistent when zooming in on one week in August 2018 (Figure 13). For the Stark intersection, it appears some spikes in $\mathrm{NO}_{2}$ values are shown at the same time for both devices, but the magnitude is different. However, for Market, each device is picking up slightly different peaks and valleys in measurement signal. Figure 14 shows Apis $\mathrm{NO}_{2}$ values tend to be higher than RAMP values but no linear relationship is evident.

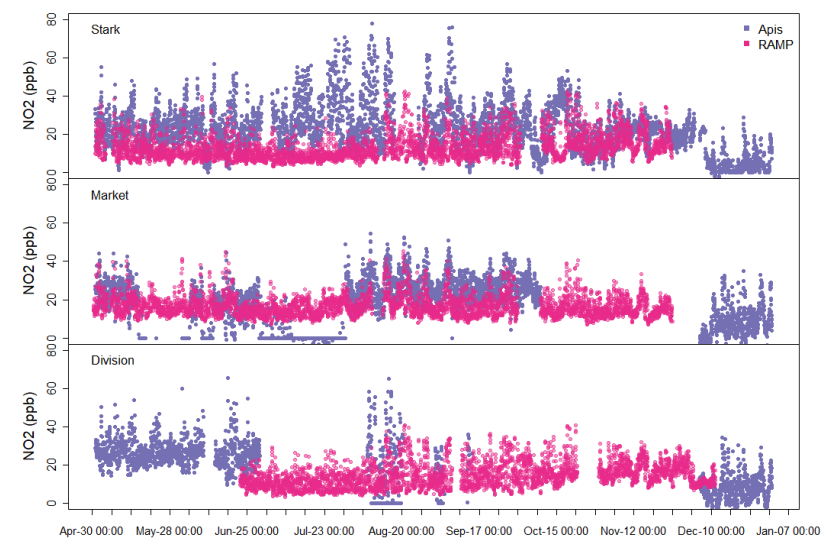

Figure 11: Hourly Apis and RAMP $\mathrm{NO}_{2}$ sensor concentrations versus time for each intersection 

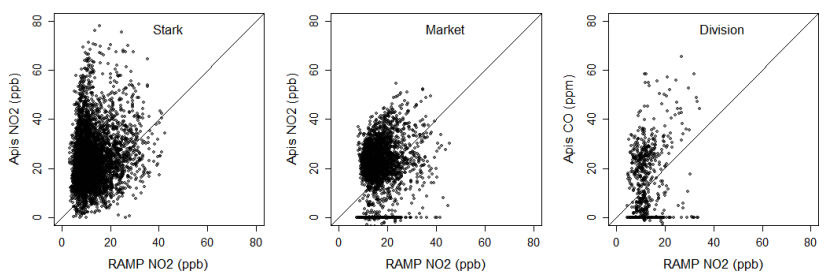

Figure 12: Apis $\mathrm{NO}_{2}$ versus RAMP $\mathrm{NO}_{2}$ concentrations for the full roadside deployment period

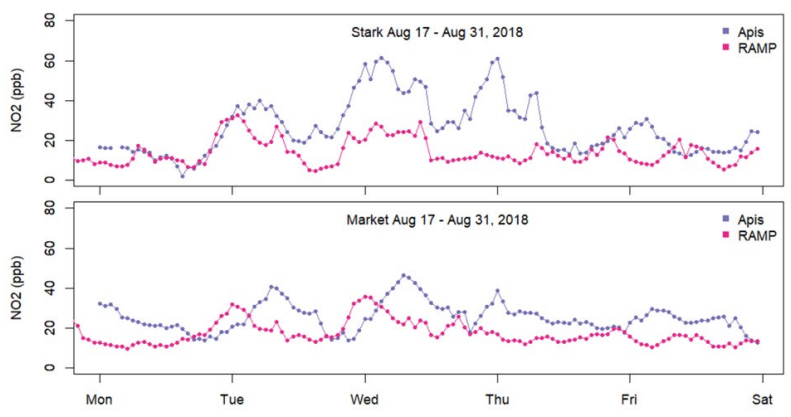

Figure 13: Apis and RAMP $\mathrm{NO}_{2}$ concentrations versus time for 1 week in August 2018 for Stark and Market intersections
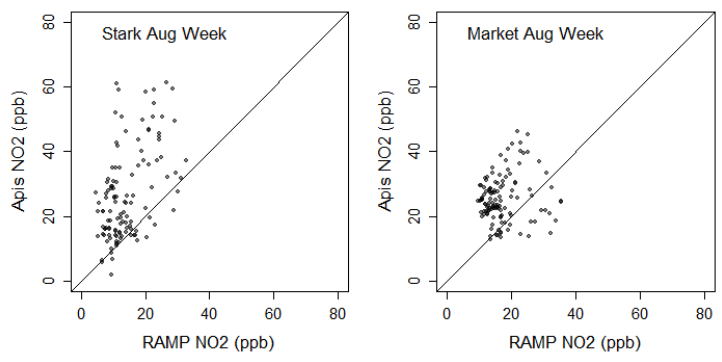

Figure 14: Apis NO2 versus RAMP $\mathrm{NO}_{2}$ concentrations for 1 week in August 2018 for Stark and Market intersections

Figures 15- 18 show the same exploratory analysis for $\mathrm{O}_{3}$ values. The relationship between Apis and RAMP $\mathrm{O}_{3}$ values differ for each intersection and by time. When zooming in on the same week in August 2018 for both Stark and Market intersections, there are distinct peaks and differences in magnitude between the different devices for $\mathrm{O}_{3}$ measurements (Figure 17). Figure 18 shows how the differences between the two sensor devices differ according to site during the same week.

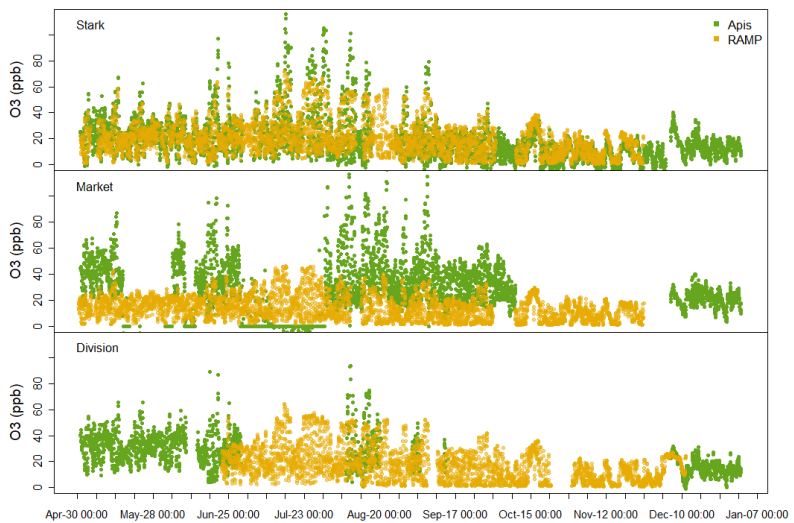

Figure 15: Hourly Apis and RAMP $\mathrm{O}_{3}$ sensor concentrations versus time for each intersection
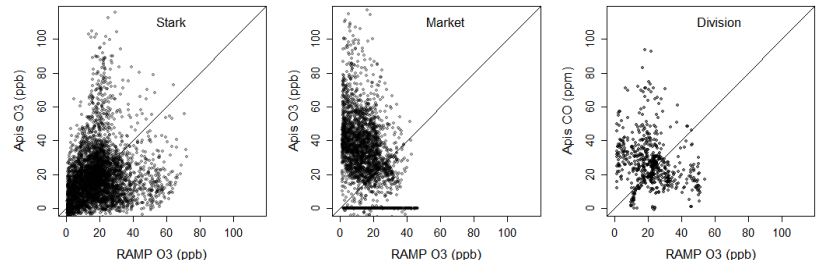

Figure 16: $\mathrm{Apis}_{3}$ versus RAMP $\mathrm{O}_{3}$ concentrations for the full roadside deployment period

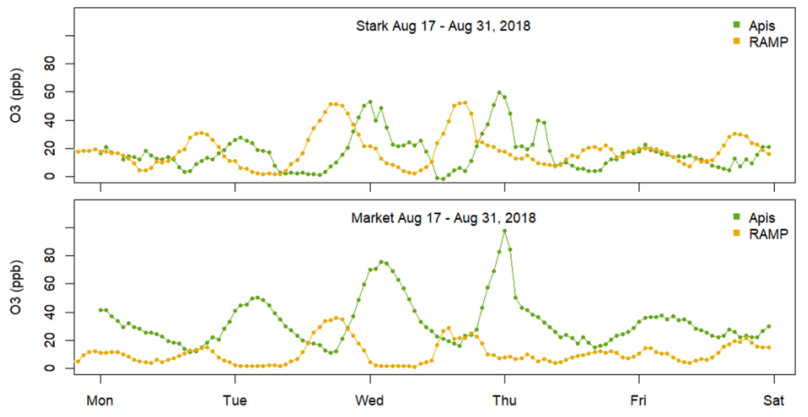

Figure 17: Apis and RAMP $\mathrm{O}_{3}$ concentrations versus time for 1 week in August 2018 for Stark and Market intersections
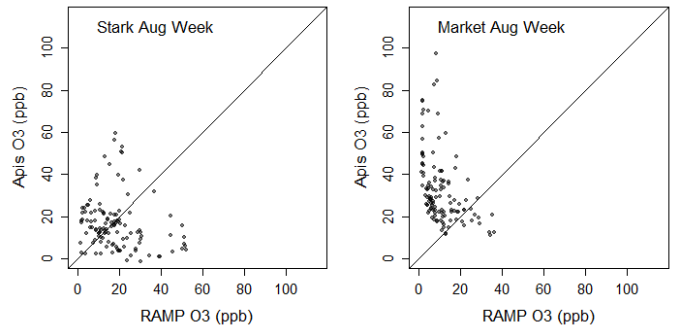

Figure 18 Apis $\mathrm{O}_{3}$ versus $\mathrm{RAMP} \mathrm{O} \mathrm{O}_{3}$ concentrations for 1 week in August 2018 for Stark and Market intersections 
Developing Best Practices for Air Quality Sensor Deployments Through Testing

Without reference instrument data co-located at each intersection, it is difficult to assess the reasons for the differences between the sensor devices. Differences could be due to calibration methods, electronics setups, original manufacturer intra- sensor variability, and more. This project is one of the first to deploy multiple types of sensors to the same location documenting an important need for further research and methods for integrating data across devices.

\section{Conclusion}

The methods used in this study covered a variety of organizational and technical considerations. In addition to documenting sensor performance, increased budget and labor needs for continued maintenance are an important finding. While the devices are designed to require as little maintenance as possible, components still needed to be replaced within months of operation. Data loss occurred across all three devices due to power and connectivity issues. These findings are consistent with past reviews noting that the cost of labor to maintain sensor networks and post-processing of data is likely to exceed costs of the sensors themselves [3].

The lifetime of lower costs sensors will not match more traditional infrastructure managed by cities. So, it is very important to consider the sustainability of the sensor components themselves to minimize electronics waste. Many small electronics are not recyclable and ubiquitous sensing has the potential to increase e-waste [3]. Incorporating modular components and the ability to upgrade devices in procurement stages can mean housing and other device and electronic components can be reused as technology improves.

The AoT Node $\mathrm{PM}_{2.5}$ and gaseous pollutant measurements showed inter-sensor variability in both lab and field deployments. Based on this testing and previous research of the Alphasense OPC N-2 $\mathrm{PM}_{2.5}$ microsensor [10], [11], correction factors are needed. Figure 6 results show a PM correction factor would be PM level dependent. For the gaseous sensors, additional calibration is needed beyond manufacturer calibrations which occur under controlled temperature and relative humidity conditions. Findings here are consistent with recommendations from other work to review data quality of each device before widely using [1].

All combined this means individual correction factors may need to be applied to each AoT Node per pollutant. To better understand the extent offsets and calibration factors are needed, additional testing in ambient environments in a broader range of pollutant concentrations is recommended.

Both the Apis and SenSevere RAMP devices applied additional calibration methods to the gaseous sensors. Differences in sensor performance especially during the roadside deployment show how these calibration methods may impact sensor measurements. Further data analysis is needed to understand when and why the devices differ across each pollutant.
SCC 2019, September, 2019, Portland, Oregon, USA

Preliminary findings do show evidence that the lower cost sensors deployed to the roadside can measure a local roadside signal in addition to urban background pollution. Establishing this pattern is key to moving forward in understanding how sensors can be used to support additional planning and assessment questions. Deployment findings show strong potential for sensors to support building awareness about air quality, documenting trends, and comparing observed air quality at locations through relative and aggregated values.

\section{ACKNOWLEDGMENTS}

Funding and support for this work were provided by NIST Replicable Smart Cities Technologies Cooperative Grant Agreement 70NANB16H258 and the Signals, Street Lighting, and Intelligent Transportation Systems (ITS) Division of the City of Portland Bureau of Transportation. The authors would also like to acknowledge the contributions from PSU STAR lab for the AoT Node testing by Philip Orlando in addition to co-authors Devin Wilde and Linda George.

\section{REFERENCES}

[1] N. Castell et al., "Can commercial low-cost sensor platforms contribute to air quality monitoring and exposure estimates?," Environ. Int., 2017.

[2] J. E. Thompson, "Crowd-sourced air quality studies: A review of the literature \& portable sensors," Trends in Environmental Analytical Chemistry. 2016.

[3] P. Kumar et al., "The rise of low-cost sensing for managing air pollution in cities," Environ. Int., vol. 75, pp. 199-205, 2015.

[4] L. Morawska et al., "Applications of low-cost sensing technologies for air quality monitoring and exposure assessment: How far have they gone?," Environ. Int., vol. 116, no. April, pp. 286-299, 2018.

[5] C. E. Catlett, P. H. Beckman, R. Sankaran, and K. K. Galvin, "Array of things: a scientific research instrument in the public way: platform design and early lessons learned," Proc. 2nd Int. Work. Sci. Smart City Oper. Platforms Eng., pp. 26-33, 2017

[6] N. Zimmerman et al., "A machine learning calibration model using random forests to improve sensor performance for lower-cost air quality monitoring," Atmos. Meas. Tech., 2018.

[7] C. Kendrick, A. Rodgers, K. Elgazzar, and B. Miles, "Recommendations for the Development \& Implementation of Distributed Sensor Networks," 2016.

[8] "PortlandMaps An Information Service by The City of Portland." [Online]. Available: https://www.portlandmaps.com/.

[9] C. M. Kendrick, P. Koonce, and L. A. . George, "Diurnal and seasonal variations of NO, NO2 and PM2.5 mass as a function of traffic volumes alongside an urban arterial," Atmos. Environ., vol. 122, no. 2, pp. 133-141, 2015.

[10] S. Sousan, K. Koehler, L. Hallett, and T. M. Peters, "Evaluation of the Alphasense optical particle counter (OPC-N2) and the Grimm portable aeroso spectrometer (PAS-1.108)," Aerosol Sci. Technol., vol. 50, no. 12, pp. 1352 $1365,2016$.

[11] L. R. Crilley et al., "Evaluation of a low-cost optical particle counter ( Alphasense OPC-N2 ) for ambient air monitoring," Atmos. Meas. Tech., vol. 11, pp. 709-720, 2018 . 